\title{
Use of unmanned aerial vehicles to supplement conventional investigation methods for underground open void stability and mitigation
}

\author{
RP Preston Golder Associates Ltd., Canada
}

J Roy Golder Associates Ltd., Canada

\begin{abstract}
As part of the scope to investigate the stability of and remediation options for a large underground open void (stope) complex at the Giant Mine Remediation Project (an inactive underground gold mine in the Northwest Territories), Golder's project team built and flew a custom unmanned aerial vehicle (UAV) in order to inspect inaccessible areas of the stope and connected development workings. The UAV surveys supplemented traditional investigation techniques including borehole-based camera surveys and LIDAR scanning (cavity monitoring system, CMS) and was successful in filling information and data gaps while reducing overall investigation costs. 3D geometry models built from UAV flight video were found to accurately reproduce the shape of the excavation when compared to incomplete CMS data collected from boreholes that intersected the void.
\end{abstract}

Keywords: unmanned aerial vehicle, drone, underground investigation, cavity monitoring system

\section{Introduction}

Many underground mining methods result in open stopes - areas which are unsafe for personnel to enter due to uncertain excavation stability. As these stopes are often adjacent to other workings, their stability performance is often of interest when planning additional underground works or long-term mine remediation. Observations of the geotechnical performance of these areas can also influence mine planning decisions for other mine areas. Conventional means of investigating their stability, including borehole camera surveys and cavity monitoring systems, are often expensive and may result in blind spots beyond the line-of-sight of the tool. Unmanned aerial vehicles (UAVs) present a rapidly emerging opportunity to supplement or replace these conventional investigation methods.

UAVs are becoming commonplace in surface mining applications (Braden 2016), and when combined with photogrammetric methods based on structure-from-motion/multiview stereography (James \& Robson 2012; Westoby et al. 2012), their ability to provide alternative views and generate centimetreaccurate 3D models is immensely valuable. Translating surface UAV technology to an underground environment presents several challenges, including lighting, lack of GPS signal to assist in navigation, and flying beyond line-of-sight. Attaching lighting to the UAV increases weight and battery load, resulting in decreased flight times. UAVs used on-surface in professional settings rely on on-board GPSs to provide extremely stable flight performance. However, when operating without GPS signal, they will tend to drift and require constant pilot input. UAVs are most useful underground when they operate beyond line-of-sight, allowing personnel to inspect otherwise inaccessible areas. This may result in difficulty maintaining radio communication when the UAV travels around corners and the intervening rock mass interferes with the signal. 


\section{Giant Mine}

Giant Mine (the site) is an inactive gold mine located approximately $5 \mathrm{~km}$ north of the centre of Yellowknife, Northwest Territories. Historically, the mine produced gold from 1948 until 1999, after which stewardship was transferred to the Indigenous and Northern Affairs Canada (INAC). INAC immediately transferred management to Miramar Giant Mine Ltd., which ceased all ore processing activities at the site but continued to mine and transport ore to the neighbouring Con Mine for processing until 2004. All mining activities ceased in July 2004, after which INAC reassumed stewardship and the Deton'Cho/Nuna Joint Venture was retained to operate and maintain the site in compliance with current regulations. This group is termed the care and maintenance contractor.

Extraction of gold was conducted with various mining methods, including cut-and-fill, shrinkage stoping, and longhole stoping. As the gold-bearing mineral at Giant Mine was predominantly arsenopyrite, a roasting process was employed to treat the ore, resulting in a by-product of arsenic trioxide dust. Considerable volumes of arsenic trioxide dust were pneumatically placed underground in previously mined stopes and purpose-mined storage chambers.

Geotechnical assessments of these stopes by Golder Associates have shown that some of these excavations pose a hazard to the project and the team had deemed these an unacceptable risk of instability in the long term. Improving the stability of these stopes is critical to the long-term remediation of the mine, and stabilisation of key underground voids has been ongoing since 2014 with placement of cemented paste backfill. As part of these remediation efforts, it is necessary to understand the geometry of the voids to be backfilled, which are often interconnected. Confirming void volumes and geometry allows engineers to design, plan, and cost backfilling activities.

During 2016, Golder conducted such investigations on the C5-09 stope complex, which consists of nine large interconnected stopes over three mining levels, each approximately 40 to $50 \mathrm{~m}$ apart. The complex is located adjacent to and underneath several underground vaults that contain arsenic trioxide dust. Currently a large void exists that, at its widest, spans approximately $30 \mathrm{~m}$ over a $70 \mathrm{~m}$ strike length and is up to $35 \mathrm{~m}$ in height. Determining the geometry of this stope complex was the focus of the 2016 investigation program. The investigation included a surface and underground drilling program and used three investigation methods - borehole camera surveys, cavity monitoring system (CMS) surveys, and UAV surveys - to confirm the geometry of the C5-09 stope complex and adjacent abutments, sills, and pillars.

\section{Investigation}

In an effort to reduce costs, the least expensive investigation methods were employed first to potentially eliminate the need for costly new boreholes.

\subsection{Borehole camera surveys}

During mining operations, exploration drilling was conducted with fan patterns drilled over regular intervals along the strike length of the orebody and into the footwall. Historical geology sections and mine level plans were reviewed and boreholes were identified that would intersect the stopes, pillars, and abutments of interest. Because of the history of the mine, the mine plans and geology sections do not always reflect the current geometry, either due to undocumented mining activities or stope wall failures. Goals of the borehole camera surveys included identifying the presence and nature of backfill and the presence and condition of abutments, pillars, and sills. One hundred and twenty-four boreholes were identified from the historical geology sections and of those, 56 were located underground and 26 were viable for borehole surveys. The rest were plugged with steel or wooden plugs, full of debris, or otherwise unserviceable. 


\subsection{Cavity monitoring system surveys}

A surveying subcontractor was retained to conduct CMS surveys using a Renishaw Cavity Auto-scanning Laser System (C-ALS) from drifts, drawpoints, and boreholes intersecting open voids. The C-ALS unit was either extended into non-entry areas or lowered into boreholes using a winch and pole system. The goal of the CMS surveys was to determine the geometry of the voids and identify potential paste backfill leakage points that could influence backfill volumes. Three locations were identified where pole-mounted CMS surveys were useful, while the remaining areas required drilling of new boreholes from underground. The cost and time associated with drilling new boreholes made it desirable to develop a custom UAV that could perform tasks similar to CMS and borehole camera surveys, i.e. checking for connections and presence and type of fill.

\subsection{Underground-specific unmanned aerial vehicle}

A project-specific UAV was developed and constructed to work in the underground environment using a $450 \mathrm{~mm}$ format frame and 10-inch propellers. The UAV was equipped with custom-designed propeller guards to protect from collisions when flying in narrow stopes as well as an on-board lighting system consisting of multi-LED infrared illuminators (Figure 1).

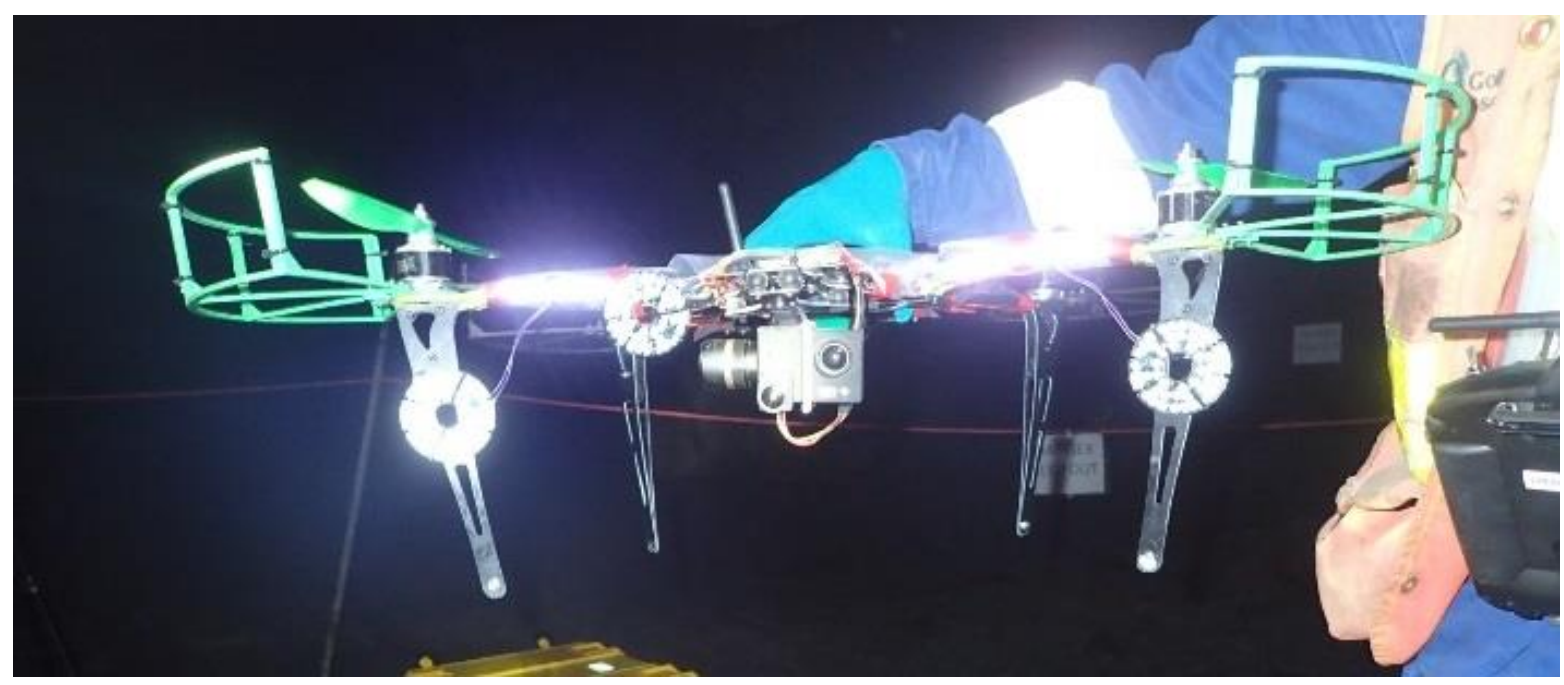

Figure 1 Frontal view of the UAV showing illuminators and propeller guards

Infrared illuminators were chosen for their low power consumption and compatibility with existing infrared cameras installed in the stope complex for geotechnical monitoring. As a result of using infrared lighting, it was necessary to modify the UAV's on-board cameras to be sensitive to infrared light. The UAV was fitted with two cameras, a highly sensitive (0.0001 lux) board camera to provide real-time video link for navigation and a second, action camera, to record high-definition video (Figure 2).

The lighting system was found to provide adequate illumination for navigation and filming up to approximately 15-20 m distance, although this was variable depending on the width of the opening with view distance reducing in wider excavations. 


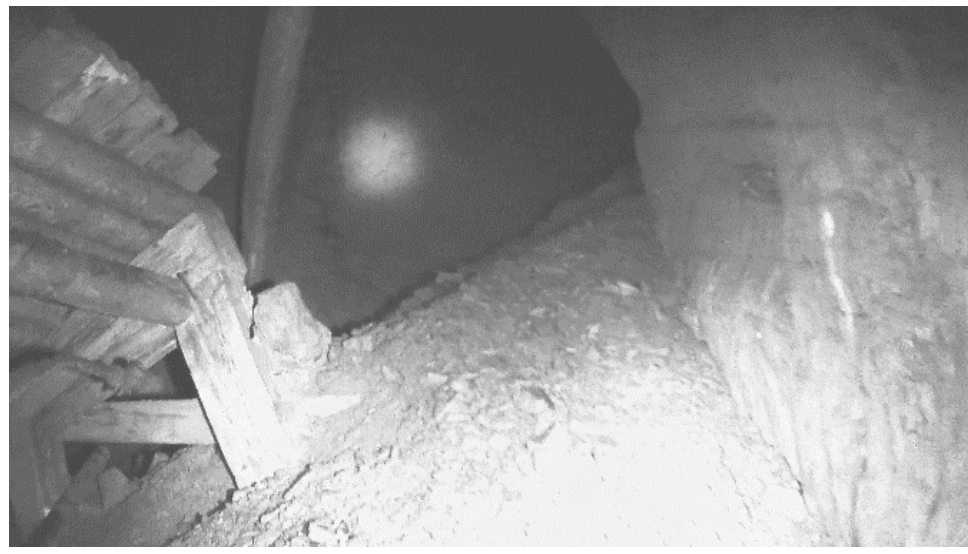

Figure 2 Video footage from flight

Both cameras were mounted to a pivoting computer-controlled support system (gimbal) to provide stable video during flight. The gimbal also allowed for tilting the camera up and down during flight. A digital video recorder was used to record the $5.8 \mathrm{GHz}$ analogue video feed from the flight camera in the event that the UAV was lost during a flight, while the data from the high-definition camera was recorded directly to an onboard micro secure digital card for retrieval at the end of the flight. The control loop of the UAV was tuned to be very docile in order to maintain stability in the GPS-denied environment. Finally, a $2.4 \mathrm{GHz}$ radio system was chosen that provided real-time data on signal strength to allow the pilot to turn back before the UAV reached the limits of the control system.

A 6,600 mAh, $14.8 \mathrm{~V}$ lithium polymer battery provided approximately 15 -minute flight times which were generally more than needed as the UAV was more commonly limited by the radio link range. The radio link range was found to be variable depending on the excavation geometry and flight path, which has a direct impact on the amount of rock intervening between the UAV and the pilot, but was generally found to be between 40 and $100 \mathrm{~m}$ during flights around a single corner.

The UAV was flown from four drifts intersecting open voids in the C5-09 complex (Figure 3). Flight one was conducted to confirm a connection between two stopes that was suspected based on irregularities in CMS data. Flights two and three were conducted in an adjacent void, with the UAV traversing between and beyond the two launch points to investigate potential connections to lower level stopes. Flight four was in a void on a lower mine level to check for open connections to the voids on the upper level.

Still images extracted from the flight videos were input into Agisoft Photoscan Professional (Agisoft 2016) and used to generate 3D geometry. The resulting model was then scaled and aligned to the CMS data for comparison.

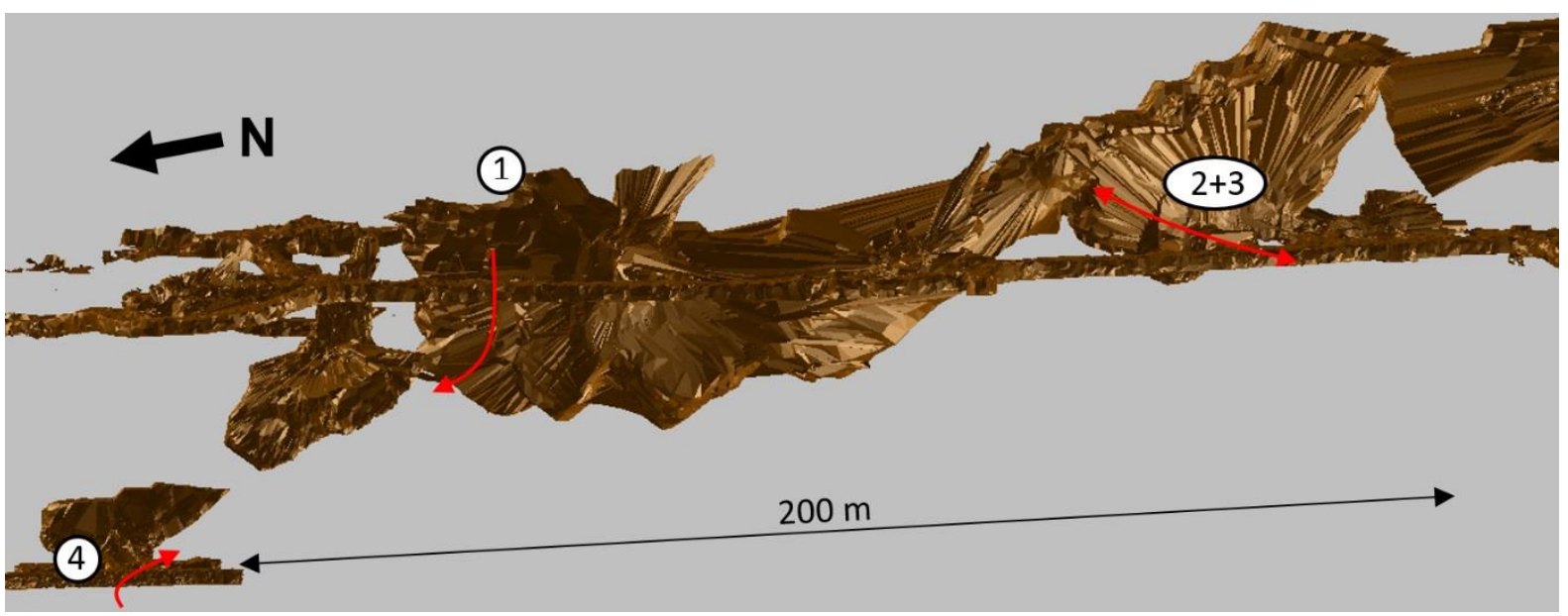

Figure 3 Section view of C5-09 Complex CMS surveys showing flight numbers and paths (red arrows), looking east 


\section{$4 \quad$ Results}

\subsection{Borehole camera surveys}

Although many of the historical boreholes identified for camera surveys could not be surveyed, the 26 that were surveyed returned valuable results that eliminated the need for drilling a number of new boreholes. Figure 4 shows a typical geology section with the length of surveyed hole highlighted in green and a screen capture from the video of the survey. By confirming intact rock along the trace of the borehole between two stopes, the presence of the sill pillar was confirmed. Borehole camera surveys confirmed the locations of two abutments, one pillar, and two sill pillars. Historical exploration holes were only drilled from the footwall side of the orebody, resulting in investigation gaps on the hanging wall side. Blind sides, as well as limited field of view and inaccessible or plugged holes in key areas, necessitated additional investigation methods including drilling new boreholes.

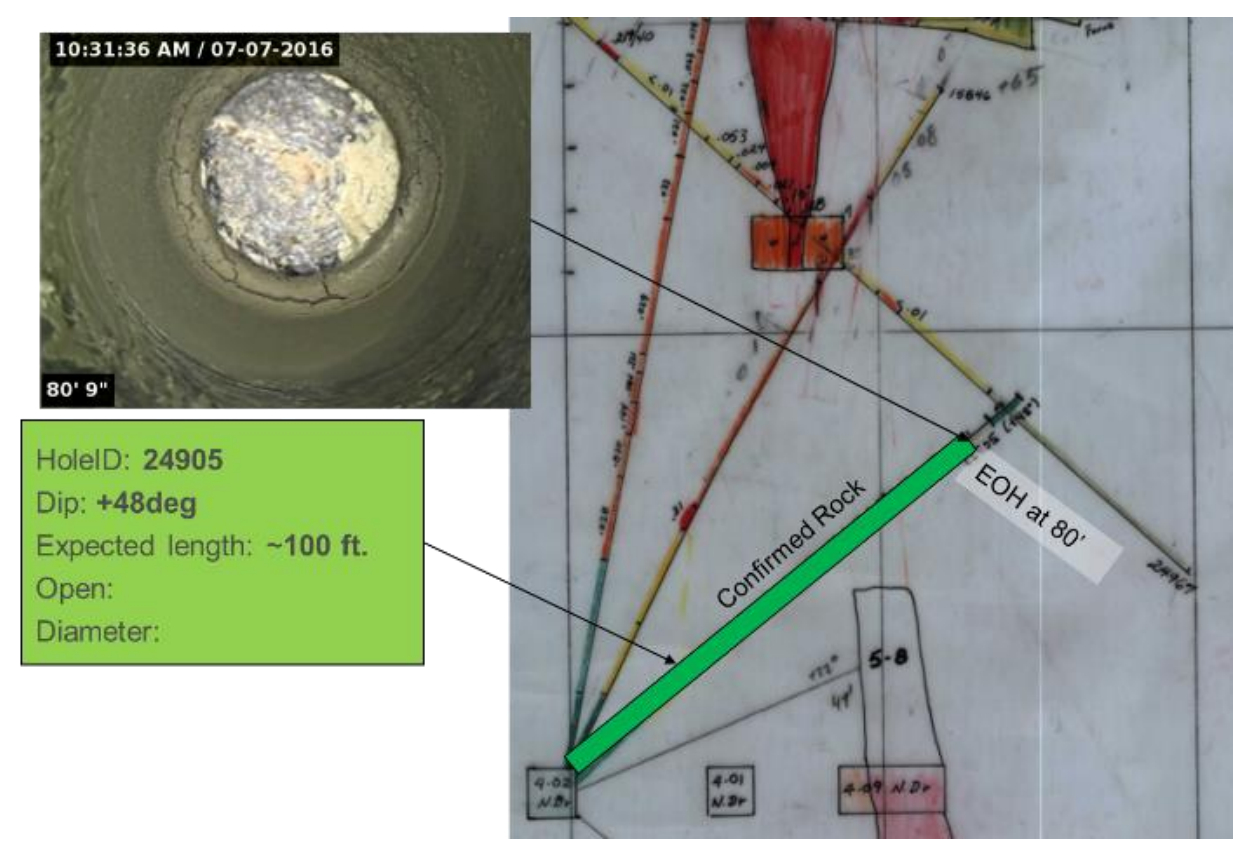

Figure 4 Results of borehole camera survey overlain on historical vertical geology cross-section

\subsection{Cavity monitoring system surveys}

Over the course of the summer investigation program, a total of nine CMS surveys were conducted. These surveys were used to confirm stope geometry and assess void fullness. These scans were also used to calculate void volumes for future backfill planning and design. Much of the geometry shown in Figure 3 was generated from these CMS scans.

\subsection{Unmanned aerial vehicle surveys}

\subsubsection{General observations}

The breakthrough at the bottom of the stope, which was suspected from CMS data, was confirmed during flight one (Figure 5). Flights two and three confirmed the presence of continuous rockfill and did not indicate sinkholes or similar evidence of vertical connections to lower stopes (Figure 6). For flight four, the UAV was flown to the end of the stope where loose, blocky rockfill was observed (Figure 7). The ability to not only confirm the presence of fill, but also assess its constituents and their particle sizes was a valuable outcome from the UAV surveys. 


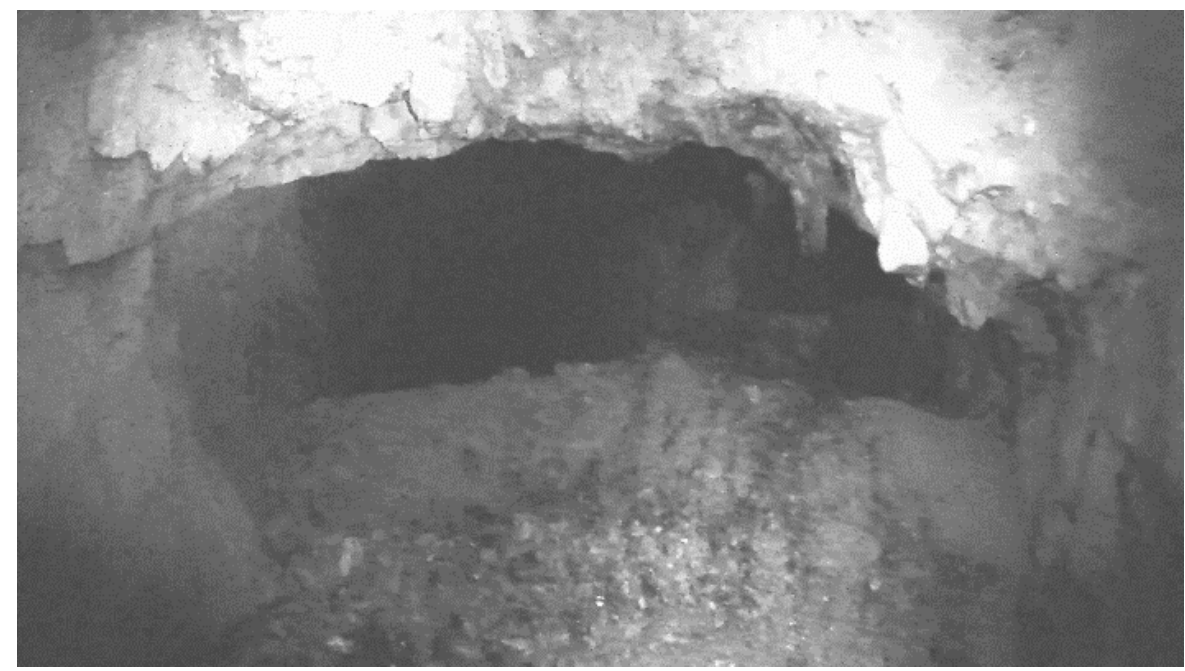

Figure 5 Breakthrough at base of stope (flight one)

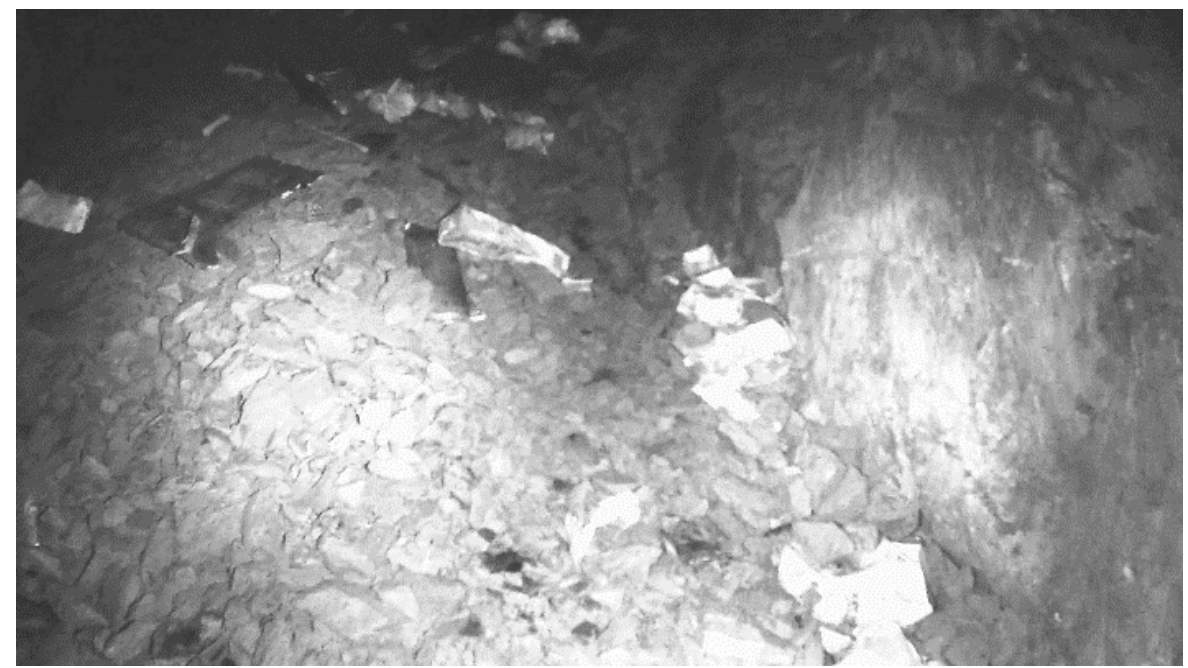

Figure 6 Rockfill and debris at base of stope (flights two and three)

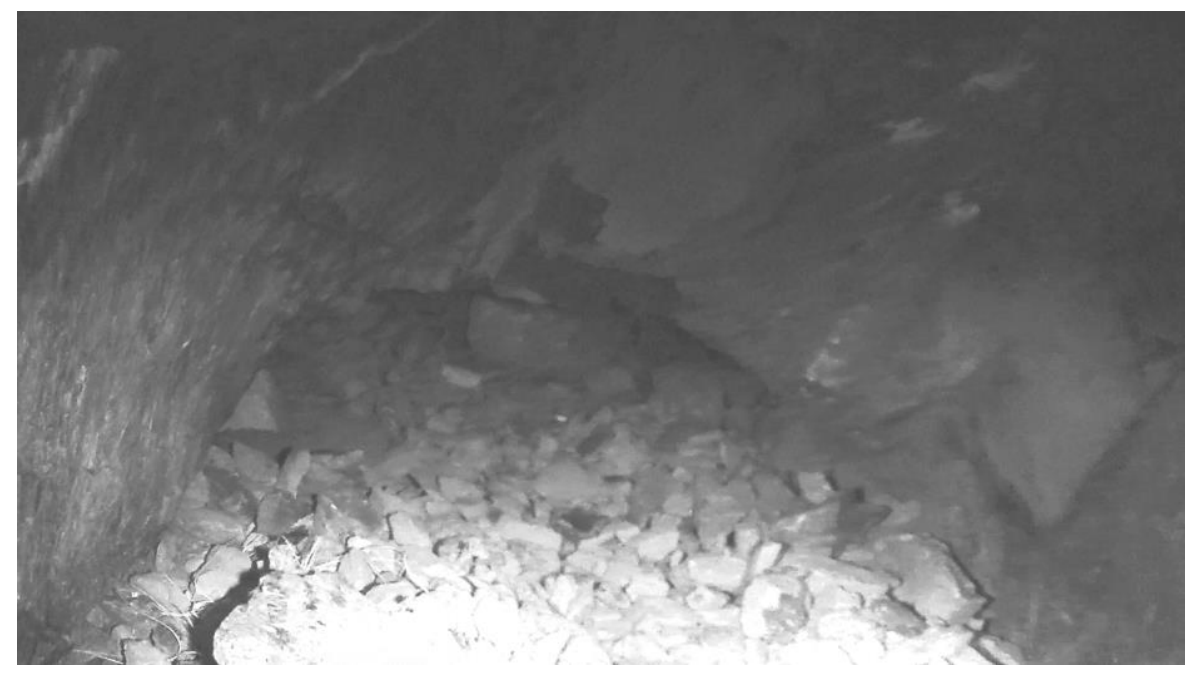

Figure 7 Blocked rockfill at end of stope (flight four) 


\subsubsection{D models from video versus cavity monitoring systems}

3D models generated using structure-from-motion photogrammetry methods were scaled and georeferenced using the lower resolution CMS data and compared to determine the accuracy of the method. Figure 8 shows the CMS solid in grey with the photogrammetry solid colour contoured based on its distance to the CMS. As shown by the legend histogram, the majority of the data agree to within $0.25 \mathrm{~m}$ with the largest errors observed at the edges of the model, where accuracy is lowest, and in the area of the breakthrough where the CMS has greatly simplified the geometry. Given the coarse resolution of the CMS scan in the area of the photogrammetry model, it is likely that the errors shown by the photogrammetry models are due to resolution discrepancies as opposed to errors in the photogrammetry model.

Depending on the geometry of a given stope, it was not always possible to build continuous 3D models from the take-off point back to landing. If the stope was too large, the on-board lights would not reach the far wall, resulting in gaps in the video that the photogrammetry software could not stitch together. In these cases, viable frames could still be extracted to build multiple separate models.

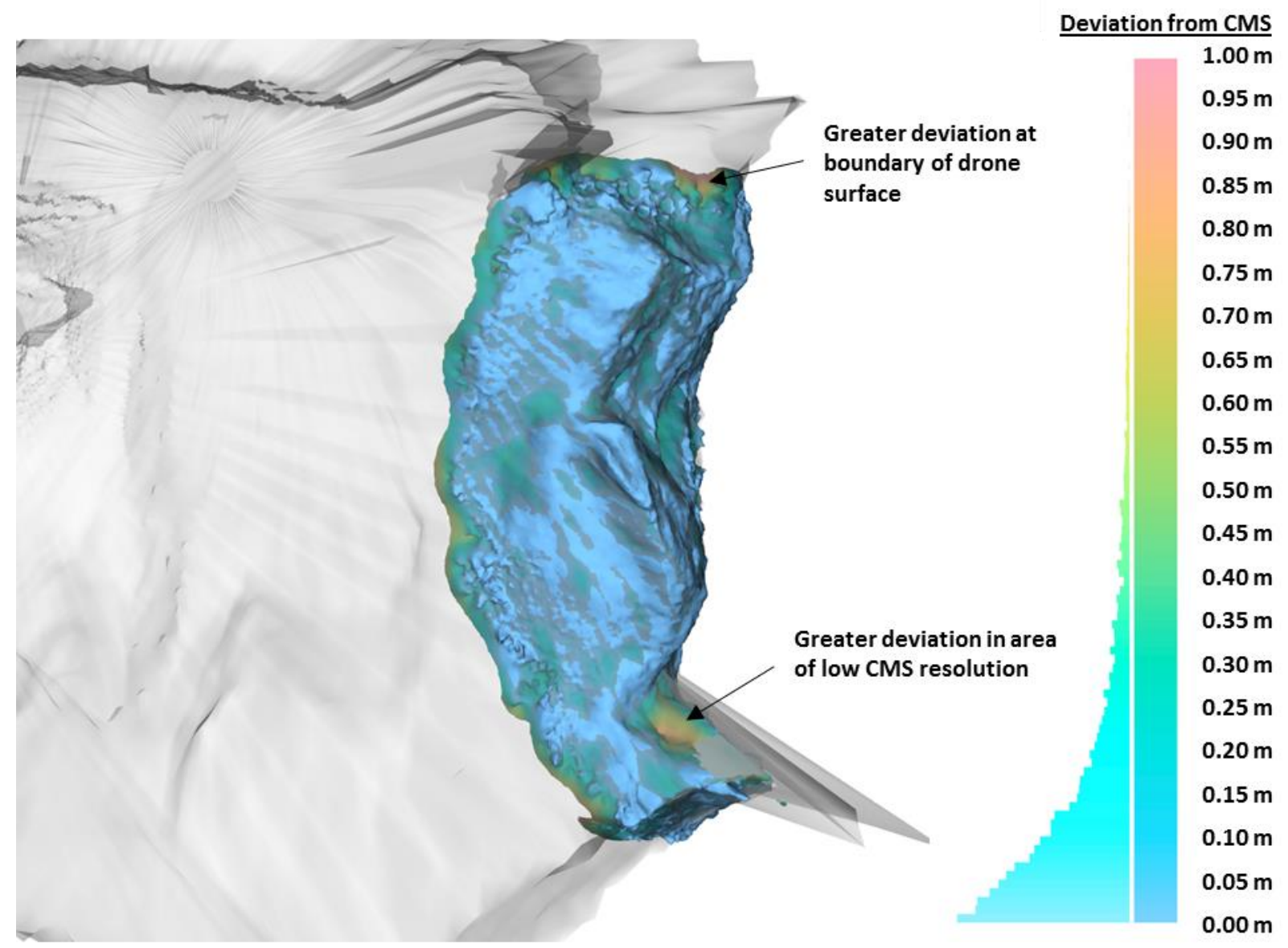

Figure 8 Comparison of photogrammetry model generated from video to the CMS survey (shown in grey)

\section{Discussion}

Borehole camera surveys are the easiest and lowest risk surveys; however, they have very limited scope due to their dependence on viable holes accessing the areas of interest. Many borehole cameras can pan and tilt and carry lighting, but viewing distance is still limited by camera resolution and light attenuation as well as line-of-sight. In addition to being relatively quick to deploy, limited training is required to operate the cameras. The greatest limitation of borehole cameras is their dependence on boreholes and the often associated need to drill camera-specific holes from surface or underground. Drilling holes for borehole camera surveys can be expensive and time consuming. 
CMS surveys are a very powerful investigative tool as they do not depend on lighting and they generate a scaled model within their line-of-sight. As CMS surveys are run from a single point, they suffer from occlusion and reduced resolution with increased distance from the tool. Post-processing the scans allows them to be georeferenced and sometimes stitched together to provide comprehensive coverage of complex stopes. Reduced scan resolution over long distances can make interpretation difficult where the 3D points are sparse and prone to spiking (large variations in position between adjacent points). The equipment is also expensive and sensitive when compared to a borehole camera and requires a specialist to operate, process and interpret.

Underground UAVs offer some of the best features of both borehole cameras and CMS. The equipment is relatively inexpensive, and surveys can be conducted rapidly. The ability to generate 3D geometry is also highly valuable, although dependent on some form of survey control within the observed area. This is less arduous than it initially appears as flight paths can be planned to proceed from areas with survey control into unknown areas. Provided well lit, quality video can be continuously captured between the points. Depending on the size of excavation, lighting can be an issue. Although extremely high-powered LEDs are available, they tend to run very hot, requiring heavy heat sinks, and can draw up to several amperes of current, further reducing flight times. The greatest advantage of UAVs is their mobility, which can reduce or eliminate blind spots in surveys of open stopes. Also, by using on-board lights, far away objects can be inspected in detail from close up. Close-up surveys come with their own risks, including signal loss, when there are obstructions between the pilot and UAV, and collisions. Despite propeller guards, collisions are still possible, and crashes often result in loss of the unit. Finally, at this time, UAVs require a specialist pilot due to the confined space they operate in and the lack of GPS signal. As collision avoidance technology improves, pilot training requirements will be reduced. Some more advanced systems with collision avoidance and scanners are already on the market. However, these units tend to cost one to two orders of magnitude more.

\section{Conclusion}

Underground mine investigations, particularly in older mines with complex history, are challenging due to data gaps and access restrictions and require multi-disciplinary approaches to be successful. The C5-09 stope investigation at Giant Mine added UAV surveys to conventional borehole camera and CMS surveys to address the project needs as quickly and efficiently as possible. Underground-specific UAVs have potential applications across almost all underground mining operations, where inaccessible areas are common and information about their stability performance would greatly assist mine planners. The ability to generate 3D geometry presents the opportunity to do real surveying and quantitatively monitor rock mass conditions. Continued advances in collision avoidance, radio links, and laser-based scanners are expected to further expand the applications of UAVs in underground mining.

\section{Acknowledgement}

The authors acknowledge the contribution of a number of individuals to the paper. Steve Miller at Deton'Cho/Nuna Joint Venture, and Chris Thomas and Chris MacInnis of INAC. Matt Heal of Golder for his assistance developing the UAV frame.

\section{References}

Agisoft 2016, Agisoft Photoscan Professional, version 1.2.6, viewed 12 April 2017, http://www.agisoft.com Braden, B 2016, 'Eyes in the high skies', Canadian Mining Journal, August 2016, pp. 38-41.

James, M \& Robson, S 2012, 'Straightforward reconstruction of 3D surfaces and topography with a camera: accuracy and geoscience application', Journal of Geophysical Research: Earth Surface (2003-2012), vol. 117, no. F3.

Westoby, MJ, Brasington, J, Glasser, NF, Hambrey, MJ \& Reynolds, JM 2012, 'Structure-from-Motion' photogrammetry: a low-cost, effective tool for geoscience applications', Geomorphology, vol. 179, pp. 300-314. 